\title{
Improvement of neurological conditions and recovery of the left ventricular ejection fraction subsequent to oxygen-ozone therapy through auto-hemoinfusion of ozonated blood
}

\author{
Sergio Pandolfi, ${ }^{1,2}$ Claudio di Giovanni, ${ }^{3}$ Eleonora Marinari, ${ }^{3}$ Marianno Franzini ${ }^{1}$ \\ ${ }^{1}$ Oxygen-Ozone Therapy Scientific Society, Gorle (BG); ${ }^{2}$ Villa Mafalda Nursing Home, Rome; \\ ${ }^{3}$ Department of Anesthesia and Intensive Care, La Sapienza University, Rome, Italy
}

\begin{abstract}
Here we present the case of a 76-year-old patient who suffered from ischemic cardiopathy and myocardial infarction in January 2014, arterial hypertension, kidney failure, Parkinsonism, vascular multinfarctual ischemic cerebropathy, cerebral ictus, neurogenic bladder, and inguinal hernia. The left ventricular ejection fraction evaluated through repeated echocardiographic examinations remained reduced to $33 \%$ from January 2014 to March 2015. The left ventricular ejection fraction, after 14 months from acute myocardial infarction and despite the coronary angioplasty and medical therapy, remained constantly reduced to $33 \%$.

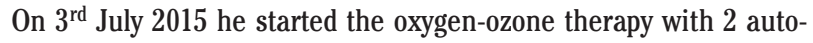
hemoinfusions of ozonated blood per week. Over the first two months of therapy we noticed a marked improvement of his heart conditions with a net reduction in asthenia and neurological status, the improvement of heart conditions was corroborated by the echocardiogram of $5^{\text {th }}$ November 2015 which showed an increase in the left ventricular ejection fraction from $33 \%$ to $50 \%$. In this case the heart function improvement of left ventricular ejection fraction was noticed only after having started the systemic oxygen-ozone therapy through large autohemoinfusion. In April 2015, before starting the oxygen-ozone therapy we assessed the patient's inguinal hernia to be inoperable due to the lapsed heart conditions, while the subsequent reassessment in December 2015, after the patient underwent oxygen-ozone therapy for
\end{abstract}

Correspondence: Sergio Pandolfi, Villa Mafalda Nursing Home, via Monte delle Gioie 5 (adiacente p.zza Vescovio), 00199 Rome, Italy.

Tel.: $+39.06 .860941 /+39.06 .86094721 /+39.06 .86094740$.

E-mail: sergiopandolfis2@gmail.com

Key words: Ischemic cardiopathy; myocardial infarction; oxygen-ozone therapy; auto-hemoinfusions; ozonated blood.

Received for publication: 26 January 2016.

Accepted for publication: 24 February 2016.

(C) Copyright S. Pandolfi et al., 2016

Licensee PAGEPress, Italy

Ozone Therapy 2016; 1:5840

doi:10.4081/ozone.2016.5840

This article is distributed under the terms of the Creative Commons Attribution Noncommercial License (by-nc 4.0) which permits any noncommercial use, distribution, and reproduction in any medium, provided the original author(s) and source are credited.
5 months, we assessed him to be operable considering the heart function improvement.

\section{Introduction}

Ozone is a gas characterized by instability, therefore it cannot be preserved but produced when needed to be used; it is naturally present in the organism of living beings, and performs several essential functions to life, it is produced by white blood cells and nowadays it is used to treat ischemic vascular pathologies since it reactivates the microcircle and increases the oxygen transfer to different organs and systems, it increases the production of energy in cells, it does not show any side effects, and does not cause any pharmacological interactions. It acts on cell metabolism and favours the elimination of toxic substances produced into cells. Oxygen-ozone therapy is a macro-therapy having a multi-organ protective action because it reactivates the micro-circle with a better oxygenation into the different body districts, it induces an increase in erythrocyte deformability and filterability with a further increase in the oxygen transfer to cells due to the increase in the production of glycerophosphate and a rightward displacement of the hemoglobin dissociation curve, it increases the mitochondrial oxidative phosphorylation, the nitric oxide release at precapillary sphincter level and the neoangiogenesis. We have constantly observed a marked improvement in his central and peripheral circulatory conditions, showing clinical outcomes which are hardly achievable with other therapies in patients suffering from ischemic cardiopathy, kidney failure, respiratory failure, ischemic vascular cerebropathy, lower limb peripheral arterial disease. Other improvements we noticed were related to the heart function in those patients suffering from ischemic cardiopathy or from myocardial infarction, with a reduction in asthenia, arrhythmia, and in vascular claudication in those patients suffering from lower limb peripheral arterial disease. In cerebrovascular pathologies and in senile dementia we notice that those patients treated with oxygen-ozone therapy show an improvement of their cognitive and mnestic faculties, of their attention, memory, motor coordination, and a reduction in micturition disorders in neurogenic bladders.

The anti-inflammatory action of ozone, leads to a reduction in the production of prostaglandins by acting on the arachidonic acid and has an antioxidant action activating the endogenous protective enzymatic functions of cells against free radicals, because it increases the transcription at DNA level of protective enzymes against free radicals.

Thanks to these characteristics it represents an essential therapy in the prevention and treatment of ischemic cardiopathy, in post-infarction rehabilitation, kidney failure, in lower limb arterial failure, in 
cerebral vascular pathologies, in ischemic ictus, and in other pathologies of the micro-circle on an ischemic and inflammatory basis.

\section{Case Report}

We present the case of a 76-year-old patient who suffered from acute myocardial infarction in January 2014, ha also suffered from Parkinsonism, arterial hypertension, chronic kidney failure, and dyslipidemia. On $15^{\text {th }}$ January 2014 he arrived at the Reception of a $2^{\text {nd }}$ level Emergency Department after a drowsiness state associated with negative dysarthria due to angina pectoris and dyspnea, the arterial pressure was $80 / 50$, the electrocardiogram (ECG) showed sinus tachycardia at $104 \mathrm{bpm}$, isolated ventricular ectopic beats, ST depression both in the front and in the back part. The ECG showed the left ventricle within the size limits, a 40\% left ventricular ejection fraction (EF), with widespread hypokinesia without marked sectionalism. The enzymes of myocardial necrosis were serum taking positive. The thorax computed tomography (CT) without contrast agent showed a pulmonary edema with multiple emery glass areas above all in lower lobes associated with pleural effusion. The brain CT was positive due to chronic ischemic vascular cerebropathy. During his stay in cardiac intensive care unit we noticed the progressive improvement of his general case history. On $21^{\text {st }}$ January 2014, an echocardiographic examination showed EF $35 \%$, widespread hypokinesia with akinesia of the basal segment of the lower wall and of the medium segments of side wall, plus a $2^{\text {nd }}$ degree diastolic dysfunction. On $28^{\text {th }}$ January 2014 the patient underwent coronary angioplasty with a direct implantation of a drug-eluting stent on the descending coronary artery and of three metal stents on the right coronary artery. On $31^{\text {st }}$ January he was discharged with the prescription of medical therapy. On $16^{\text {th }}$ April 2014 the patient underwent an ECG that showed an increase in the mass index of left ventricle, while segmentary asynergy documented a severe EF global and longitudinal systolic impairment of $33 \%$, an increased diastolic filling pressure and a mild mitral valve insufficiency. The subsequent echocardiographic examinations performed on $31^{\text {st }}$ October 2014, 29 ${ }^{\text {th }}$ January 2014, and $20^{\text {th }}$ April 2014 respectively, the ECG showed and EF of 33\%. In July 2015 the patient started the oxygen-ozone therapy through large autohemoinfusion of ozonated blood, during the sessions $240 \mathrm{mg}$ of blood were taken through venous access with needle-cannula, the blood was collected in a closed-circuit SANO3 bag specifically certified, and then mixed with $200 \mathrm{cc}$ of $\mathrm{O}_{2} / \mathrm{O}_{3}$ gaseous mixture at $35 \mathrm{mg}$ through a MED95 Computerized Photometric System medical device [Multiossigen srl., Gorle (BG), Italy] and subsequently re-infused into the vein.

In the first two months from the beginning of the therapy the patient already showed a marked improvement of his heart conditions with a reduction in asthenia and a marked improvement of neurological conditions.

The improvement of heart conditions was corroborated by the ECG performed on $5^{\text {th }}$ November 2015 , which showed an increase in the left ventricular ejection fraction from 33\% to 50\%. In April 2015, before starting the oxygen-ozone therapy we assessed the patient's inguinal hernia to be inoperable due to the lapsed heart conditions, while the subsequent reassessment in December 2015, after the patient underwent oxygen-ozone therapy for 5 months, we assessed him to be operable considering the heart and kidney function improvement.

\section{Discussion}

Ozone is a gas characterized by instability, it is naturally present in the organism and in the atmosphere. Its therapeutic applications have been unacknowledged for a long time. Its administration in the organism, even if at the very beginning shows an oxidizing action, leads to an initial and transitory oxidative stress, the blood and plasma redox potential is made up of several high performing systems cooperating with each other and effective in opposing the initial oxidizing action of ozone that performs a real preconditioning from oxidative damage, its therapeutic concentrations do not exceed the blood antioxidant potential. ${ }^{1-3}$

The ozone put in contact with plasma reacts instantly with the antioxidant systems [above all uric acid, ascorbic acid, glutathione (GSH), cysteine, albumin] therefore only a small part of the initial dose reacts with polyunsaturated fats, which are mainly present at the three albumin hydrophobic cavities level (Eq. 1):

$$
-\mathrm{R}-\mathrm{CH}=\mathrm{CH}-\mathrm{R}+\mathrm{H}_{2} \mathrm{O}+\mathrm{O}_{3} \rightleftharpoons 2 \mathrm{RCHO}+\mathrm{H}_{2} \mathrm{O}_{2}
$$

Therefore the potential ozone energy is transferred to two main messengers, such as $\mathrm{H}_{2} \mathrm{O}_{2}$, and the aldehyde 4-hydroxynonenal molecules (4HNE) molecules and trans-4-hydroxyhexanal (HHE). Given the high reactivity of ozone such reactions take place in a few seconds, and usually a few minutes of contact between blood and oxygen-ozone mixture are enough for the ozone to exhaust completely and for the oxygen to saturate the hemoglobin system and dissolve into plasma. The antioxidant systems are mainly reintegrated in the 20 min following the exposure to the gaseous mixture.

The deionized $\mathrm{H}_{2} \mathrm{O}_{2}$, enters mainly all hematic cells quickly and makes some changes: i) erythrocytes: glycolysis activation, increase in intracellular concentrations of ATP and 2,3-diphosphoglycerate. This turns into a rightward displacement of the hemoglobin dissociation curve, which, therefore, releases oxygen to peripheral tissues more easily; ${ }^{3}$ ii) leucocytes: the phagocytic activity of neutrophils increases. In lymphocytes and macrophages the IkB intracellular patterns are activated, $\mathrm{IkB}$ is one of the $\mathrm{kB}$ nuclear transcription factor components. Trimer, which is degraded in the proteasome into heterodimer p50-p65, can activate the transcription of more than 100 genes. Interleukins and some acute-phase proteins are produced. Furthermore interferon and tumor necrosis factor are produced; 4 iii) platelets: platelet-derived growth factor polypeptide, transforming growth factor $\beta 1$ and growth factors are produced.

Aldehydes, reacting with GSH, carnosine and, above all, albumin, are carried into the different body tissues. The toxicity of 4HNE and HHE is opposed by compensatory mechanisms such as detoxification, dilution and excretion; since they are recognized as oxidizing agents, the body responds by producing superoxide dismutase, heme oxygenase, glucose6-phosphate dehydrogenase and other powerful antioxidants. Moreover, aldehydes stimulate the production of endothelial nitric oxide synthase (eNOS), ${ }^{5}$ improving the peripheral perfusion and the tissue oxygenation. The auto-hemoinfusion of ozonated blood proved to be safe and well tolerated by patients. Furthermore, it has been pointed out in a randomized clinical study on 140 patients, 70 of which underwent oxygen-ozone therapy and the other 70 representing the control group, that the hematochemical parameters showed a reduction in reactive oxygen metabolytes [300 \pm 10.1 Carratelli units (U.CARR.) after 12 months compared to an initial value of $380 \pm 10.4$ U.CARR., $P<0.05$ ] and an increase in the values of the plasma biological antioxidant potential $(2100 \pm 34.8$ umol/vitamin $\mathrm{C}$ after 12 months compared to the initial value of $1610 \pm 36.2, P<0.05$ ) in treated patients compared to the control group. ${ }^{6}$

These data demonstrate that the auto-hemoinfusion of ozonated blood plays a crucial role in reducing the oxidative stress by endogenously stimulating the production of antioxidant enzymes.

The oxygen-ozone therapy is a safe method, having no side effects, which does not cause any allergic reactions and is currently used to treat several pathologies (lower limb obliterative arteriopathy, cutaneous ulcers, atrophic maculopathy of retina, ${ }^{6}$ vascular pathologies on ischemic and degenerative basis, brain pathologies on vascular and 
degenerative basis, etc. ${ }^{2,7}$ The studies carried out on animals, show that among the mice that underwent myocardial ischemia, the groups showing ischemic areas and the best heart function indexes were those to which an oxygen-ozone mixture was administered. $5,8,9$ According to the in vivo studies, in 2008 the ACCLAIM trial was published $; 10$ a randomized double-blind clinical study in which NYHA II-IV patients were administered ozonated blood with the Celacade system (Vasogen Inc., Mississauga, Canada), involving small blood quantities (10-20 mL) exposed to large concentrations of ozone, then processed and warmed up to 42 by means of ultraviolet radiation and subsequently injected again in gluteus. The study, which investigated the correlation between the administration of this therapy called Nonspecific immune modulating and the clinical evaluation items such as death due to any cause, hospitalization due to cardiological causes, the need of an intravenous therapy for the decompensation, etc. In any case results have been in favor of the ozone therapy, even though the therapeutic procedure carried out was incomplete and the large auto-hemoinfusion was not performed, which is suitable for all organ, micro-circle, and ischemic pathologies, in fact the scientific community has challenged the use of a method like the Celacade (Vasogen Inc.) for the ozone therapy, since the very high concentrations used on an exiguous blood sample exceed the redox potential of plasma and the heat and ultraviolet rays denature most of corpuscular components. ${ }^{11-14}$

The effects of the acute administration of an oxygen/ozone $\left(\mathrm{O}_{3}\right)$ mixture on the myocardial tissue damage after an ischemic event have been observed. ${ }^{8}$ The study was performed on rats that underwent acute myocardial ischemia and reperfusion. Animals have been treated with an oxygen-ozone mixture of 100,150 and $300 \mathrm{mg} / \mathrm{kg}$ insufflated intraperitoneally an hour before the ischemic damage.

The size of the infarction has been measured and also the necrosis and tissue damage markers, the nitrotyrosine, CD68, CD8, CD4 and the caspase-3. Results have highlighted a smaller size of the infarction area in those rats that were pre-treated with the insufflation of the oxygenozone mixture and the parallel decrease in the nitrotyrosine tissue levels and in the inflammation (CD68) and immune response (CD8 and CD4) markers. These data indicate that the damage associated with ischemia and myocardial reperfusion can be opposed by a pre-treatment through the systemic administration of the oxygen-ozone gaseous mixture.

Recent studies showed that after a myocardial infarction the levels of endothelial progenitor cells (EPCs) reduced into the myocardium. These cells derive from the bone marrow and aim at mobilizing, migrating, and differentiating into endothelial cells in situ and creating a cell reserve able to make up for endothelial damage. Strategies aimed at increasing the EPCs into an ischemic heart seem to improve the neovascularization in the ischemic tissue, and could improve the myocardial blood flow and reduce the ischemic damage. An experimental study on animals ${ }^{8}$ pointed out that the oxygen/ozone protects the heart from acute myocardial infarction thanks to the local increase in the eNOS activity and to the recruitment of endothelial progenitor cells.

The purpose of this study has been to establish whether a mixture of oxygen/ozone $\left(\mathrm{O}_{2} / \mathrm{O}_{3}\right)$ protects the heart from acute myocardial infarction through the local involvement of eNOS and of EPCs. The SpragueDawley male rats underwent $25 \mathrm{~min}$ of occlusion and $2 \mathrm{~h}$ of reperfusion of the front descending coronary artery. The $\mathrm{O}_{2} / \mathrm{O}_{3}$ mixture was insufflated for $30 \mathrm{~min}$ intra pericardium before the ischemic/reperfusion (I/R) procedure with the following doses: 100,150 and $300 \mu \mathrm{g} / \mathrm{kg}$. We have carried out the measurements of the size of the myocardial infarction (IS) and the myocardial immunohistochemistry with EPCs search. For these cells we have evaluated in the infarcted tissue the immunoreactivity to CD34 and CD117/c-kit. Moreover we have monitored the heart eNOS. The I/R in rats treated with $\mathrm{O}_{2}$ produced an IS as percentage of the area at risk equal to $51 \pm 5 \%$. The $\mathrm{I} / \mathrm{R}$ in rats treated with $\mathrm{O}_{2} / \mathrm{O}_{3}$ mixture showed a reduced IS (for example, the IS/rheumatoid arthritis for $150 \mu \mathrm{g} / \mathrm{kg}$ $0_{2} / 0_{3}$ was $35 \pm 2.1 \% ; \mathrm{P}<0.01$ vs $\mathrm{O}_{2}$ ). The $\mathrm{O}_{2} / \mathrm{O}_{3}$ cardioprotection occurred together with an increase in the immunopositive particles per area of CD34 and CD117/c-kit. The increase in the number of these markers has been associated with an increase in the expression of heart eNOS as dosed by immunohystochemistry. The $\mathrm{O}_{2} / \mathrm{O}_{3}$ mixture protects heart from acute myocardial infarction through the local increase in the eNOS expression/activity and the consequent EPC recruitment.

The levels of endothelial progenitor cells after a myocardial infarction are reduced in the heart. EPCs derive from the bone marrow, migrate and differentiate into endothelial cells to make up for endothelial damage.

The $\mathrm{O}_{2} / \mathrm{O}_{3}$ administration before an attack of acute ischemia-reperfusion leads to a greater eNOS-dependent recruitment of EPCs into the infarcted myocardium and to a reduction in the infarction necrosis area.

\section{Conclusions}

The oxygen-ozone therapy through large auto-hemoinfusion protects the heart of patients suffering from ischemic cardiopathy and it is essential in the rehabilitation of those patients who had an acute myocardial infarction as clinical outcomes and experimental evidence pointed out.

\section{References}

1. Bocci V. Is it true that ozone is always toxic? The end of a dogma. Toxicol Appl Pharmacol 2006:216:493-504.

2. Bocci V, Zanardi I, travagli V. Oxygen/ozone as medical gas mixture. A critical evaluation of the various methods clarifies positive and negative aspects. Med Gas Res 2011;1:6.

3. Bocci V, Borrelli E, Travagli V, Zanardi I. The ozone paradox: ozone is a strong oxidant as well as medical drug. Med Res $\operatorname{Rev} 2009 ; 29$ : 646-82.

4. Viebahn-Hänsler R. The use of ozone in medicine: mechanisms of action; Munich - May 23-25, 2003. Available from: http://www.o3 center.org/Articles/TheUseofOzoneinMedicine.pdf

5. Di Filippo C, Luongo M, Marfella R, et al. Oxygen/ozone protects the heart from acute myocardial infarction through local increase of eNOS activity and endothelial progenitor cells recruitment. Naunyn Schmiedebergs Arch Pharmacol 2010;382:287-91.

6. Borrelli E, Bocci V. Visual improvement following ozonetherapy in dry age related macular degeneration; a review. Med Hypothesis Discov Innov Ophthalmol 2013;2:47-51.

7. Elvis AM, Ekta JS. Ozone therapy: a clinical review. J Nat Sci Biol Med 2011;2:66-70.

8. Di Filippo C, Marfella R, Capodanno P, et al. Acute oxygen-ozone administration to rats protects the heart from ischemia reperfusion infarct. Inflamm Res 2008;57:445-9.

9. Merin 0. Ozone administration reduces reperfusion injury in an isolated rat heart model. J Card Surg 2007;22:339-42.

10. Torre-Amione G. Results of a non-specific immunomodulation therapy in chronic heart failure (ACCLAIM trial): a placebo-controlled randomised trial. Lancet 2008;371:228-36.

11. Melchart D. Immunomodulation through autohemotherapy in chronic heart failure. Forsch Komplementmed 2008;15:230.

12. Bocci V. Non-specific immunomodulation in chronic heart failure. Lancet 2008;371:2083; author reply 2084.

13. Sliwa K, Ansari AA. Immunosuppression as therapy for congestive heart failure. Lancet 2008;371:184-6.

14. Fildes JE, Shaw SM, Yonan N, Williams SG. Non-specific immunomodulation in chronic heart failure. Lancet 2008;371:2083; author reply 2084 